\title{
Design Space Exploration of Initial Structural Design Alternatives via Artificial Neural Networks
}

\author{
Ozan Yetkin ${ }^{1}$, Arzu Gönenç Sorguç ${ }^{2}$ \\ ${ }_{1,2}$ Middle East Technical University, Department of Architecture, Building Science \\ 1,2\{oyetkin|arzug\}@metu.edu.tr
}

\begin{abstract}
Increasing implementation of digital tools within a design process generates exponentially growing data in each phase, and inevitably, decision making within a design space with increasing complexity will be a great challenge for the designers in the future. Hence, this research aimed to seek potentials of captured data within a design space and solution space of a truss design problem for proposing an initial novel approach to augment capabilities of digital tools by artificial intelligence where designers are allowed to make a wise guess within the initial design space via performance feedbacks from the objective space. Initial structural design and modelling phase of a truss section was selected as a material of this study since decisions within this stage affect the whole process and performance of the end product. As a method, a generic framework was proposed that can help designers to understand the trade-offs between initial structural design alternatives to make informed decisions and optimizations during the initial stage. Finally, the proposed framework was presented in a case study, and future potentials of the research were discussed.
\end{abstract}

Keywords: design space, objective space, structural design, artificial intelligence, machine learning, optimization

\section{INTRODUCTION}

The conceptual structural design phase of an architectural project is a very critical stage since decisions made during that stage affects the whole process. Thus, it is very crucial for a designer to consider possible instances within the solution space so that the alternative selected by the designer would satisfy aimed objectives. Since conceptual structural design involves multi-objective criteria, it is impossible to process and analyze a vast amount of possible alternatives via the data-crunching capacity of a human brain. Therefore, this research aims to propose a novel approach in computer-aided analysis and design with the implementation of machine learning algorithms in which users can interact with the algorithm during the process of modeling and explore design space effectively by using the interactive computational methods.

\section{Background}

Background of this research has shown that the initial design phase is very important since the whole process is started to be defined in these stages. Therefore, it is crucial for designers to consider as much 
option and variable as possible to optimize the design process. Unfortunately, the data processing capacity of a human brain is limited to a portion of the enormous possibility of space; but processing data via machine learning can extend that portion and as a consequence, have a potential to augment the design space exploration process.

For instance, in a research conducted by Brown and Mueller (2017), a study was done on the design space catalog which is a collection of different options for designers to choose and how it can go beyond by designing with data. As a method, they reviewed the developments in four main categories that are interaction, automation, simplification, and visualization. Then they discussed that human-computer interaction should be integrated into early stage design and even in the initial brainstorming phase. Finally, they concluded that humancomputer collaboration can harness the power of computational processes together with the creative capacities of designers to generate workflows that answer to the complexity of the architecture.

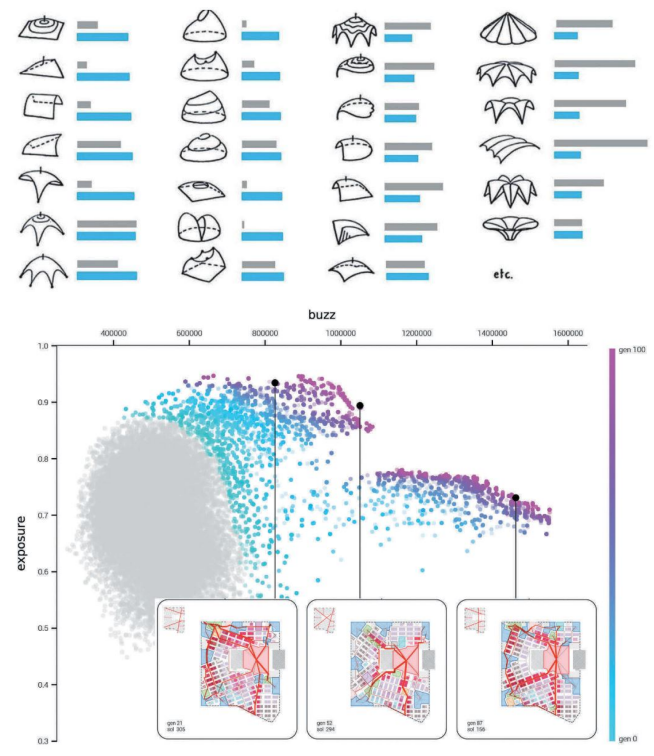

Also, in another research done by Villaggi et al. (2017), researchers proposed a novel design space approach to be used in generative space planning in architecture. The model they developed was based on a data structure that allows subdivision and merges operations on a floor plan controlled by a smaller set of input parameters. Also model includes evaluation for the performance of generated floor plans using a set of congestion metrics that allows them to be optimized by a genetic algorithm. Researchers also presented a set of guidelines and methods for analyzing and visualizing the quality of the model within the solution space (Figure 2). Finally, researchers concluded that the developed model could allow designers to break free of standard rules and explore a broader range of design possibilities.

\section{Research Problem and Objective}

The problem of this research was mainly based on the idea that the complexity of computer-aided analysis and design models are increasing exponentially and it is getting more complicated for a designer to consider numerous design alternatives within the design space. Besides, review on previous research showed that computational methods and machine learning algorithms have a potential to enhance the design space exploration as Alpaydin denoted this in his book as "Machine learning will help us make sense of an increasingly complex world. Already we are exposed to more data than what our sensors can cope with, or our brains can process." (2016).

The main objective of this research is implementing machine learning models into the computational design tools and allowing designers to harness the data processing capacity of computers to explore design space more effectively. Another objective is that since previous research within the field of computational design mainly focuses on optimization and search for 'the solution', this research aims to propose a framework which derives correlations between design space and objective space to allow the designer to explore multiple solutions by controlling aimed structural performance.
Figure 1

An example design space (Brown and Mueller, 2017)

Figure 2

Quality metrics visualized within the solution space (Villaggi et al., 2017) 
Figure 3

Different design

alternatives

generated by

different

parameters

Figure 4

Flowchart of the research method

\section{RESEARCH DESIGN}

Since every design process is unique and aim of the research was not to search for the single optimum solution for a specific problem but to learn the solution space of a user-defined problem, the main research question was whether initial design alternatives proposed by the designer used as a starting point for the algorithm or not. Also, another research question was to test machine learning algorithms to see if they can extract features and derive correlations within multi-objective criteria to make an evaluation.

The hypothesis of this research was based on the idea that machine learning algorithms have the potential to be implemented to the initial structural design phase as a pier to enhance design space exploration. Secondly, since structural elements have measurable quantitative criteria, metrics for evaluating the performance of initial structural design alternatives can be used for multi-objective optimization of structural models. Last but not least, since structural elements have rule-based criteria, machine learning models have the potential to extract features and derive correlations in data to make accurate evaluations.

\section{Material of the Research}

The first research material is the initial geometry data from the designer modelled within the conceptual structural design phase, which should have parameters to generate new design alternatives. For this research, Karamba's sample parametric structural design models were used (Preisinger, 2016). The second research material is the structural performance data composed of the total mass of the structure and maximum displacement, calculated with Karamba parametric engineering components (Preisinger and Heimrath, 2014). The last research material is artificial neural networks (ANN), which was fed with input data composed of design variables and output data comprising structural simulation data. In this study, Crow artificial neural network component developed by Felbrich (2016) was used.
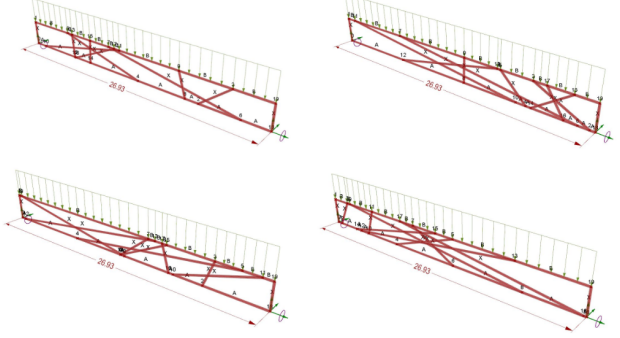

\section{Method of the Research}

As a method, listed parameters that control the initial structural geometry were initialized within a gene pool to generate new geometries in a randomized and unbiased manner (Rutten, 2013). After creating new geometries by using Galapagos genetic solver (Rutten, 2010) that shuffles input variables, two sets of data that are maximum displacement and the total mass of structure were calculated with finite element method by Karamba components for each design alternatives. Since two sets of recorded quantitative data for maximum displacement and the total mass have different ranges ( 0.03 to 0.45 and 4175 to 9138 respectively), both were normalized and remapped between 0 to 1 just as input data in order each data feature to affect learning model equally. Then both input data from a gene pool that controls the geometry and output data from the simulation that gives the score for displacement and total mass were assigned to an artificial neural network engine to evaluate (Figure 4).

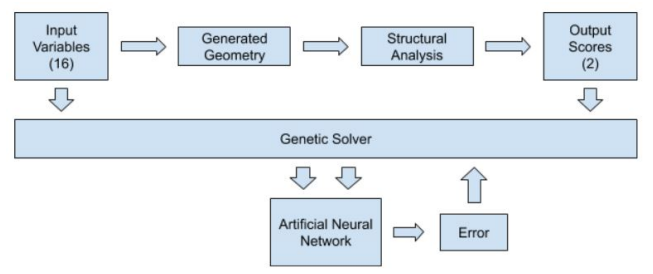



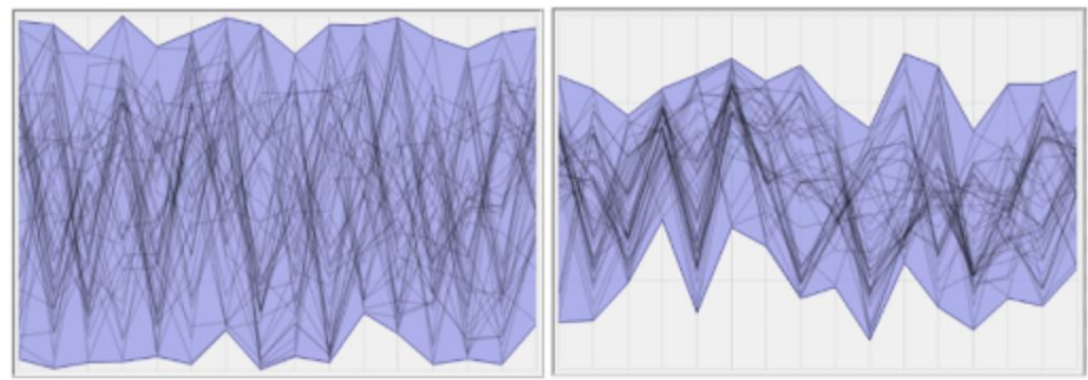

Figure 5

Area chart of 16

input variables which defines the

design space for

generations 1, 5, 25 and 50
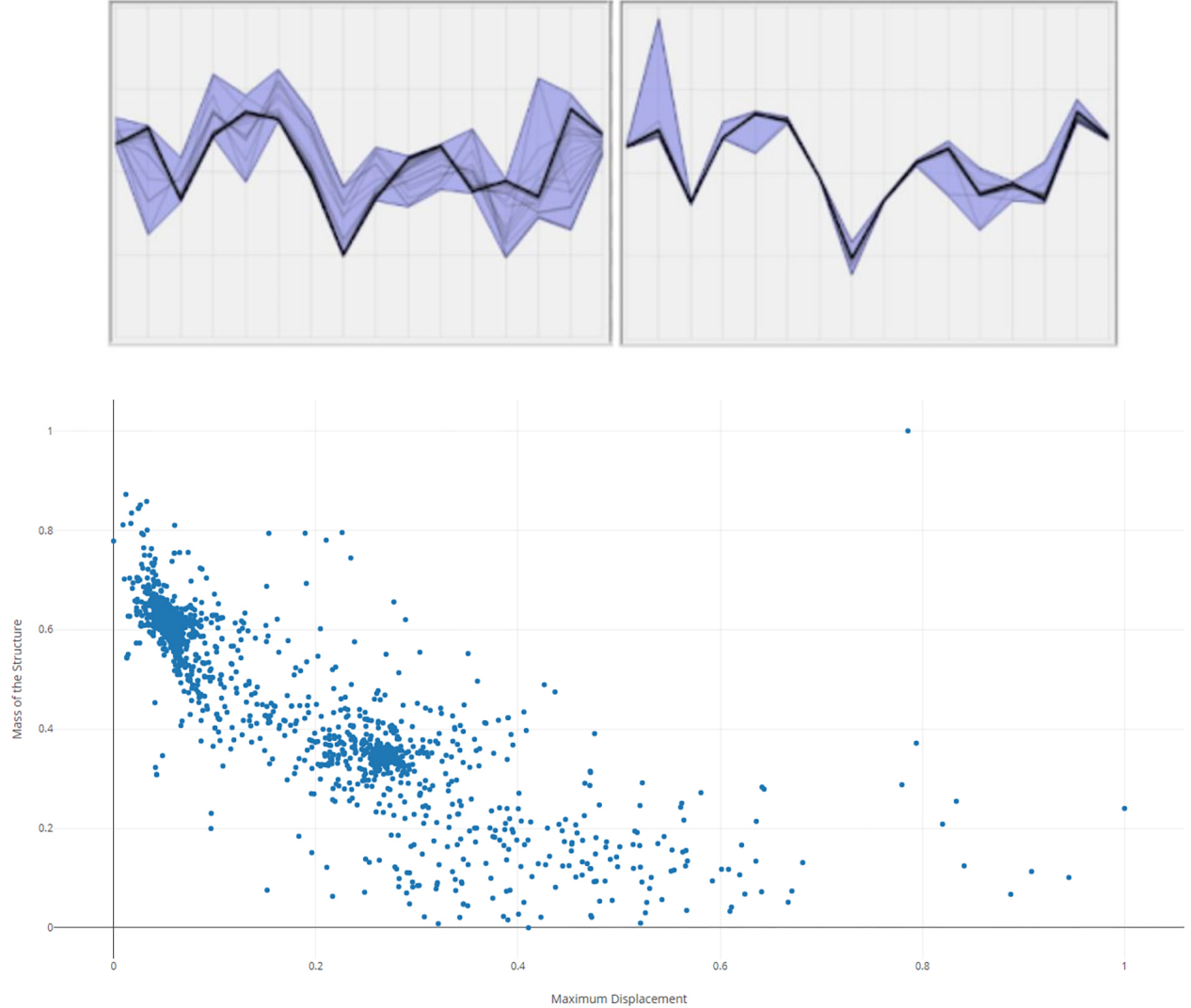

Figure 6

Scatter plot of 2 output variables which defines the objective space: mass of the structure and maximum displacement 
Figure 7

The network architecture of 4 by 4 ANN and 8 by 8 ANN for 16 inputs and 2 outputs

Figure 8

Line graph of mean squared error over iterations

Figure 9

The extended generic framework proposed for future studies

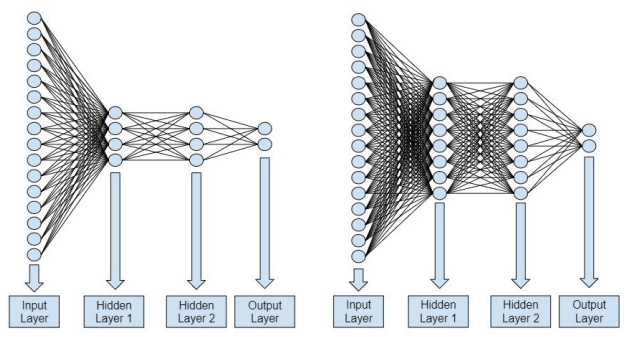

ANALYSIS AND RESULTS

Sample parametric model for optimization of truss diagonals from Karamba was used for data capturing process, as mentioned in the material of research. This sample parametric model has 16 input variables (start and end point of 8 truss diagonals) that control the whole geometry and 2 selected output variables that are the mass of the structure and maximum displacement. Data collected by randomizing these 16 variables within a gene pool in a randomized manner by using Galapagos genetic solver and recording 2 output data for each design alternative. Finally, mean squared error of a trained artificial neural network was calculated with the formula below where $N$ is the number of data points, $f_{i}$ is the value returned by the model and $y_{i}$ is the actual value for each data point $i$.

$$
M S E=\frac{1}{N} \sum_{i=1}^{N}\left(f_{i}-y_{i}\right)^{2}
$$

After data were collected, initial training of the artificial neural network was conducted with 350 and 1150 different design alternatives, and it was observed that the error of artificial neural network decreases as more data were given. After that, Galapagos evolutionary solver was used to generate different design alternatives by genetic shuffling and fed the artificial neural network with input and output in each iteration to minimize the mean squared error of the ANN. After 5264 iterations, mean squared error rate of the artificial neural network was decreased to 0.11614 when ANN with four by four hidden layer architecture was used. After that, the same data of 5264 different design alternatives were given to an ANN with eight by eight hidden layers and mean squared error was decreased to 0.063562 . Finally, the trained artificial neural network with the mean squared error rate of 0.0636 was used to predict diagonal support positions of a truss when different scores for mass and displacement were given, and it was observed that predicted positions were accurate. 


\section{CONCLUSION AND FUTURE PROJECTIONS}

To conclude, this research has shown that machine learning algorithms have the potential to be implemented to the initial structural design phase to catalyze design space exploration. Also, the mean squared error rate has proven that machine learning algorithms can be trained to extract features and derive correlations within multi-objective criteria to make accurate and precise predictions. Yet, this research must be extended by implementing the same methodology to different design examples to prove the hypothesis that the proposed learning model is sufficient to learn the solution space of various problems. Also, the study should be implemented with more variables within the design space such as material type, thickness, number of supporting elements, load type and quantity and so on to test if the learning model can still learn the design space by using correlations between inputs and outputs. Therefore, a generic framework is proposed for future studies (Figure 9), based on the initial study conducted within the scope of this research.

\section{REFERENCES}

Alpaydin, E. 2016, Machine Learning: The New Al, MIT Press

Brown, N. and Mueller, C. 2017 'Designing with Data: Moving Beyond the Design Space Catalog', ACADIA 2017

Preisinger, C. and Heimrath, M. 2014, 'Karamba-A Toolkit for Parametric Structural Design', Structural Engineering International, 24(2), pp. 217-221

Rutten, D. 2010 'Evolutionary Principles Applied to Problem Solving Using Galapagos', AAG10, Vienna

Rutten, D. 2013, 'Galapagos: On the Logic and Limitations of Generic Solvers', Architectural Design, 83(2), pp. 132-135

Villaggi, L., Zhao, D. and Benjamin, D. 2017 'Beyond Heuristics: A Novel Design Space Model for Generative Space Planning in Architecture', ACADIA 2017

[1] http://www.felbrich.com/projects/Crow/Crow.html

[2] https://www.karamba3d.com/examples/ 